\title{
Synthesis of PID Controller with Guaranteed Stability Margin for a Class of Unstable MIMO Systems
}

\author{
T. S. Chang
}

\begin{abstract}
A systematic synthesis procedure is presented to obtain a Proportional+Integral+Derivative (PID) controller for a class of linear time-invariant multi-input multi-output (MIMO) plants, which achieves the closed-loop stabilization with guaranteed stability margin. Numerical examples are given to illustrate its feasibility.
\end{abstract}

Keywords- Stabilization and tracking, PID control, integral action, stability margin.

\section{INTRODUCTION}

The Proportional+Integral+Derivative (PID) controllers are widely used in applications. Recently, rigorous PID synthesis methods are explored (please see [1] and its references). In [1], sufficient conditions are given to guarantee the stability of MIMO systems with PID controllers, and several plants classes that admit PID controllers were identified. By extending the work in [1], a systematic synthesis procedure is presented in [2] to design PID controllers with guaranteed stability for given stable MIMO systems. A sufficient condition is also given for the existence for such a controller.

The goal of this paper is to study closed-loop stabilization with guaranteed stability margins using PID-controllers. Along the same line as that in [2], some results in [1] are extended to include the systematic synthesis procedures of PID controller design for certain classes of stable or unstable MIMO systems. Numerical examples are given to illustrate its feasibility.

Section II shows the main results, where sufficient conditions are given for the existence of PID controllers. Section III presents the systematic synthesis procedures for synthesizing PID controllers. Some examples are used to illustrate their feasilbity. Section IV gives a short discussion.

\section{SUFFICIENT CONDITIONS}

Let $\mathbb{C}, \mathbb{R}, \mathbb{R}_{+}$denote complex, real, positive real numbers. The extended closed right-half complex plane is $\mathcal{U}=$ $\{s \in \mathbb{C} \mid \operatorname{Re}(s) \geq 0\} \cup\{\infty\} ; \mathbf{R}_{\mathbf{p}}$ denotes real proper rational functions of $s ; \mathbf{S} \subset \mathbf{R}_{\mathbf{p}}$ is the stable subset with no poles in $\mathcal{U} ; \mathcal{M}(\mathbf{S})$ is the set of matrices with entries in $\mathbf{S} ; I_{n}$ is the $n \times n$ identity matrix. The $H_{\infty}$-norm of $M(s) \in \mathcal{M}(\mathbf{S})$ is $\|M\|:=\sup _{s \in \partial \mathcal{U}} \bar{\sigma}(M(s))$, where $\bar{\sigma}$ is the maximum singular value and $\partial \mathcal{U}$ is the boundary of $\mathcal{U}$. We drop $(s)$ in transfer-matrices such as $G(s)$ wherever this causes no confusion. We use coprime factorizations over $\mathbf{S}$;

The author is with the Department of Electrical and Computer Engineering, University of California, Davis, CA 95616. (emails: chang@ece.ucdavis.edu) i.e., for $G \in \mathbf{R}_{\mathbf{p}}{ }^{n_{y} \times n_{u}}, G=Y^{-1} X$ denotes a left-coprimefactorization (LCF), where $X, Y \in \mathcal{M}(\mathbf{S})$, $\operatorname{det} Y(\infty) \neq 0$.

Consider the linear time-invariant (LTI) MIMO unityfeedback system $\operatorname{Sys}(G, C)$ shown in Fig. 1, where $G \in$ $\mathbf{R}_{\mathbf{p}}{ }^{m \times m}$ is the plant's transfer-function and $C \in \mathbf{R}_{\mathbf{p}}{ }^{m \times m}$ is the controller's transfer-function. Assume that $\operatorname{Sys}(G, C)$ is well-posed, $G$ and $C$ have no unstable hidden-modes, and $G \in \mathbf{R}_{\mathbf{p}}{ }^{m \times m}$ is full (normal) rank. We consider the realizable form of proper PID-controllers given by (1), where $K_{p}, K_{i}, K_{d} \in \mathbb{R}^{m \times m}$ are the proportional, integral, derivative constants, respectively, and $\tau \in \mathbb{R}_{+}$[3]:

$$
C_{\text {pid }}=K_{p}+\frac{K_{i}}{s}+\frac{K_{d} s}{\tau s+1} .
$$

For implementation, a (typically fast) pole is added to the derivative term so that $C_{p i d}$ in (1) is proper.

Definition 2.1: a) $\operatorname{Sys}(G, C)$ is said to be stable iff the transfer-function from $(r, v)$ to $(y, w)$ is stable. $b) C$ is said to stabilize $G$ iff $C$ is proper and $\operatorname{Sys}(G, C)$ is stable. $\triangle$

The problem addressed here is the following: Suppose that $h \in \mathbb{R}_{+}$is a given constant. Can we find a PIDcontroller $C_{p i d}$ that stabilizes the system $\operatorname{Sys}\left(G, C_{p i d}\right)$ with a guaranteed stability margin, i.e., with real parts of the closed-loop poles of the system $\operatorname{Sys}\left(G, C_{p i d}\right)$ less or equal to $-h$ ? It is clear that this goal is not achievable for some plants. Furthermore, even when it is achievable, it may be possible to place the closed-loop poles to the left of a shiftedaxis that goes through $-h$ only for certain $h \in \mathbb{R}_{+}$.

To investigate the problem, let define

$$
\begin{gathered}
\hat{s}:=s+h, \text { or } \quad s=: \hat{s}-h \\
\text { and } \hat{G}(\hat{s}):=G(\hat{s}-h) .
\end{gathered}
$$

The problem is reduced to the equivalent question: Whether the system $\hat{G}(\hat{s})$ can be stabilized by the controller $\hat{C}_{p i d}$ in $\hat{s}$-space defined as

$$
\hat{C}_{p i d}(\hat{s}):=K_{p}+\frac{K_{i}}{\hat{s}-h}+\frac{K_{d}(\hat{s}-h)}{\tau(\hat{s}-h)+1} .
$$

In the following two propositions, let $G(s)$ have no transmission-zeros in $\mathcal{U}$ other than the real-axis blockingzeros stated in the propositions, which can be the zero at infinity. However, $G(s)$ may have any number of transmissionzeros in the stable region. The poles of $G(s)$ are completely arbitrary, except that we assume $G(s)$ has no pole at $s=0$ if there is a zero close to the origin. We will use the two step design method by first obtaining the Proportional+Derivative (PD) controller, and then adding the Integral (I) portion to form the overall PID controller as in [1]. 
Proposition 1: (Systems with a large RHP zero)

Let $G(s)$ have no pole at $s=0$. It has one blocking-zero at $s=z_{1}$, where $0<z_{1} \leq \infty$ and $z_{1} \in \mathbb{R}$. Let $h \geq 0$. Choose any $k_{p}>0, a_{1}>h$ and $0<\tau_{1}<\frac{1}{h}$.

$$
\begin{aligned}
& \hat{G}=\hat{Y}^{-1} \hat{X}=\left(\frac{\tilde{x}_{1}}{\tilde{y}_{1}} \hat{G}^{-1}\right)^{-1}\left(\frac{\tilde{x}_{1}}{\tilde{y}_{1}} I\right), \text { where } \\
& \tilde{x}_{1}=1-(\hat{s}-h) / z_{1}, \quad \tilde{y}_{1}=(\hat{s}-h)+a_{1} .
\end{aligned}
$$

Define

$$
\begin{gathered}
Y(\infty):=\lim _{s \rightarrow \infty} \frac{\left(1-s / z_{1}\right)}{\left(s+a_{1}\right)} G^{-1}(s) \\
\Psi_{1}(\hat{s}):=\tilde{y}_{1} \hat{Y} Y(\infty)^{-1}\left[\frac{\tau_{1}(\hat{s}-h)+1}{\tau_{1}(\hat{s}-h)+k_{p}}\right]-(\hat{s}-h) I
\end{gathered}
$$

If $\left\|\Psi_{1}(\hat{s})\right\|<z_{1} \leq \infty$, then for any $\alpha$ satisfying

$$
\alpha>\left(1-\left\|\Psi_{1}(\hat{s})\right\| / z_{1}\right)^{-1}\left(\left\|\Psi_{1}(\hat{s})\right\|+h\right)
$$

the PD controller $C_{1}(s)$ below stabilizes $G(s)$ with guaranteed stability margin specified by $h$ :

$$
C_{1}(s)=Y(\infty) \frac{\alpha}{\left(1+\alpha / z_{1}\right)}\left[k_{p}+\frac{\left(1-k_{p}\right) \tau_{1} s}{\tau_{1} s+1}\right]
$$

Proof: From (10), we can get

$$
\hat{C}_{1}(\hat{s})=\frac{\alpha}{\left(1+\alpha / z_{1}\right)}\left[\frac{\tau_{1}(\hat{s}-h)+k_{p}}{\tau_{1}(\hat{s}-h)+1}\right] Y(\infty),
$$

Choose the RCF for $\hat{C}_{1}(\hat{s})$ as

$$
\hat{C}_{1}=\hat{N}_{c} \hat{D}_{c}^{-1}=\left(\hat{C}_{1}\right)(I)^{-1} .
$$

Define

$$
\begin{gathered}
y_{\alpha}:=(\hat{s}-h)+\alpha \\
\hat{M}_{1}:=\hat{X} \hat{C}_{1}+\hat{Y}=\left(\frac{\tilde{x}_{1}}{y_{\alpha}} \alpha I+\frac{\tilde{y}_{1}}{y_{\alpha}} \hat{Y} \alpha \hat{C}_{1}^{-1}\right) \frac{y_{\alpha}}{\tilde{y}_{1}} \alpha^{-1} \hat{C}_{1} \\
=\left(I-\frac{\left(1+\alpha / z_{1}\right)(\hat{s}-h)}{y_{\alpha}} I+\frac{\tilde{y}_{1}}{y_{\alpha}} \hat{Y} \alpha \hat{C}_{1}^{-1}\right) \frac{y_{\alpha}}{\tilde{y}_{1}} \alpha^{-1} \hat{C}_{1} \\
\hat{M}_{1}=\left[I-\frac{\left(1+\alpha / z_{1}\right)}{y_{\alpha}} \Psi_{1}(\hat{s})\right] \frac{y_{\alpha}}{\tilde{y}_{1}} \alpha^{-1} \hat{C}_{1}
\end{gathered}
$$

Since

$$
\lim _{(\hat{s}-h) \rightarrow \infty} \Psi_{1}(\hat{s})=a_{1} I,
$$

$\Psi_{1}(\hat{s}) \in \mathcal{M}(\mathbf{S})$. With the choice of $\alpha, a_{1}, k_{p}$ and $\tau_{1}$, $\hat{M}_{1}(\hat{s})$ is unimodular. Thus, $\hat{C}_{1}(\hat{s})$ stabilizes $\hat{G}(\hat{s})$, and $C_{1}(s)$ stabilizes $G(s)$ with guaranteed stability margin specified by $h$.

Proposition 2: (Systems with two large RHP zeros) Let $G(s)$ have no pole at $s=0$. It has two blocking-zeros $0<z_{1} \leq z_{2} \leq \infty$, and $z_{i} \in \mathbb{R}$. Let $h \geq 0$. Choose any $a_{1}>h, a_{2}>h$ and $k_{2}>h$.

$$
\begin{gathered}
\hat{G}=\hat{Y}^{-1} \hat{X}=\left(\frac{\tilde{x}}{\tilde{y}} \hat{G}^{-1}\right)^{-1}\left(\frac{\tilde{x}}{\tilde{y}} I\right), \\
\tilde{x}=\left(1-(\hat{s}-h) / z_{1}\right)\left(1-(\hat{s}-h) / z_{2}\right), \\
\tilde{y}=\left((\hat{s}-h)+a_{1}\right)\left((\hat{s}-h)+a_{2}\right) .
\end{gathered}
$$

Define

$$
\begin{aligned}
Y(\infty):=\lim _{s \rightarrow \infty} \frac{\left(1-s / z_{1}\right)\left(1-s / z_{2}\right)}{\left(s+a_{1}\right)\left(s+a_{2}\right)} G^{-1}(s) \\
=\lim _{(\hat{s}-h) \rightarrow \infty} \hat{Y}(\hat{s})
\end{aligned}
$$

Define

$$
\Psi_{2}(\hat{s}):=\frac{\tilde{y}}{\left((\hat{s}-h)+k_{2}\right)} \hat{Y} Y(\infty)^{-1}-(\hat{s}-h) I
$$

For any $\alpha>0$ and $\beta>0$ satisfying

$$
\begin{gathered}
\alpha+1 / z_{1}<1 / h, \\
\beta+1 / z_{2}<1 / h, \\
\eta / \rho<1 / h, \\
\frac{(\rho-\eta h)}{\left(1-\left(\alpha+1 / z_{1}\right) h\right)\left(1-\left(\beta+1 / z_{2}\right) h\right)}<\left\|\Psi_{2}(\hat{s})\right\|^{-1},
\end{gathered}
$$

where

$$
\begin{gathered}
\rho=\alpha+\beta+2\left(1 / z_{1}+1 / z_{2}\right) \\
\eta=\alpha \beta+\alpha / z_{2}+\beta / z_{1},
\end{gathered}
$$

the PD controller $C_{2}(s)$ below stabilizes $G(s)$ with guaranteed stability margin specified by $h$ :

$$
C_{2}(s)=\rho^{-1}\left[k_{2}+\frac{\left(1-k_{2} \tau_{2}\right) s}{\tau_{2} s+1}\right] Y(\infty), \text { where } \tau_{2}=\frac{\eta}{\rho} .
$$

Proof: From (30), we can get

$$
\hat{C}_{2}(\hat{s})=\left[\frac{(\hat{s}-h)+k_{2}}{\rho+\eta(\hat{s}-h)}\right] Y(\infty),
$$

Choose the RCF for $\hat{C}_{2}(\hat{s})$ as

$$
\hat{C}_{2}=\hat{N}_{c} \hat{D}_{c}^{-1}=\left(\hat{C}_{2}\right)(I)^{-1} .
$$

Define

$$
\begin{gathered}
y_{\alpha}:=1+\left(\alpha+1 / z_{1}\right)(\hat{s}-h), \\
y_{\beta}:=1+\left(\beta+1 / z_{2}\right)(\hat{s}-h), \\
\hat{M}_{2}:=\hat{X} \hat{C}_{2}+\hat{Y}=\left(\frac{\tilde{x}}{y_{\alpha} y_{\beta}} I+\frac{\tilde{y}}{y_{\alpha} y_{\beta}} \hat{Y} \hat{C}_{2}^{-1}\right) \frac{y_{\alpha} y_{\beta}}{\tilde{y}} \hat{C}_{2} \\
=\left(I-\frac{(\hat{s}-h)(\rho+\eta(\hat{s}-h))}{y_{\alpha} y_{\beta}} I+\frac{\tilde{y}}{y_{\alpha} y_{\beta}} \hat{Y} \hat{C}_{4}^{-1}\right) \frac{y_{\alpha} y_{\beta}}{\tilde{y}} \hat{C}_{4} \\
=\left[I+\frac{(\rho+\eta(\hat{s}-h))}{y_{\alpha} y_{\beta}} \Psi_{2}(\hat{s})\right] \frac{y_{\alpha} y_{\beta}}{\tilde{y}} \hat{C}_{2}
\end{gathered}
$$

Since

$$
\left.\Psi_{2}(\hat{s})\right|_{(\hat{s}-h) \rightarrow \infty}=\left(a_{1}+a_{2}-k_{2}\right) I,
$$

$\Psi_{2}(\hat{s}) \in \mathcal{M}(\mathbf{S})$. With the choice of all parameters, $\hat{M}_{2}(\hat{s})$ is unimodular. Thus, $\hat{C}_{2}(\hat{s})$ stabilizes $\hat{G}(\hat{s})$, and $C_{2}(s)$ stabilizes $G(s)$ with guaranteed stability margin specified by $h$.

To get the PID-controller, we use the two-step design procedure mentioned in [1]. Let $C_{p d}(s)$ be a PD-controller stabilizing $G(s)$ with guaranteed stability margin specified by 
$h$, which can be either $C_{1}(s)$ or $C_{2}(s)$ in previous propositions. Equivalently, $\hat{C}_{p d}(\hat{s})$ is a PD-controller stabilizing $\hat{G}(\hat{s})$, and

$$
\hat{H}_{p d}(\hat{s}):=\hat{G}\left(I+\hat{C}_{p d} \hat{G}\right)^{-1} \in \mathcal{M}(\mathbf{S}) .
$$

Finding an I-controller for $\hat{H}_{p d}(\hat{s})$ is the special case in [2] by letting $\hat{K}_{p}=0$ and $\hat{K}_{d}=0$. Once $\hat{C}_{I}(\hat{s})$ is found, the controller $\hat{C}_{\text {pid }}(\hat{s})=\hat{C}_{p d}(\hat{s})+\hat{C}_{I}(\hat{s})$ is a PID-controller stabilizing $\hat{G}(\hat{s})[4]$.

Proposition 3: (PID-controller) Let $G(s)$ have no pole at $s=0$. Define

$$
\gamma(h):=\left\|\frac{\hat{H}_{p d}(\hat{s}) H_{p d}(0)^{-1}-I}{\hat{s}-h}\right\|^{-1} .
$$

For any given PD-controller $C_{p d}(s)$ stabilizing $G(s)$ with guaranteed stability margin specified by $h(\geq 0)$, if we can find an $\alpha$ such that

$$
h<\alpha<\gamma(h)-h,
$$

the PID-controller

$$
C_{p i d}(s):=C_{p d}(s)+C_{I}(s),
$$

where

$$
C_{I}(s)=\frac{(\alpha+h) H(0)^{-1}}{s},
$$

stabilizes $G(s)$ with guaranteed stability margin specified by $h$.

Remark: If $h=0$, the results are reduced to those corresponding results in [1].

\section{PID CONTROLLER SYNTHESIS}

The propositions in the previous section not only give us sufficient conditions, but also suggest a systematic synthesis procedure for PID-controller design. We will use two single input single output (SISO) examples to illustrate the systematic procedure, since the design for MIMO systems follows the same procedure but with more computation involved.

Example 3.1: Given the plant transfer function

$$
G(s)=\frac{(s+5)\left(s^{2}+8 s+32\right)}{(s+2)(s-8)\left(s^{2}-12 s+40\right)},
$$

it is an unstable plant with one RHP zero at $z_{1}=\infty$. We use Proposition 1 to design a PID-controller.

Consider $h=1.5$. Choose arbitrarily $\tau_{1}=0.05, k_{p}=$ 1 and $a_{1}=3$. From (7), we get $Y(\infty)=1$. From (8), we compute $\left\|\Psi_{1}(\hat{s})\right\|=31.61$, which leads to the lower bound 33.11 of $\alpha$ from (9). Choose $\alpha=34$. Then, the PDcontroller in (10) can be calculated. The closed-loop poles are $-2.41+i 2.08,-2.41-i 2.08,-5.59+i 21.04,-5.59-$ $i 21.04,-20.0$, which satisfy the given $h=1.5$.

To add the I-controller from Proposition 3, we calculate $\hat{H}_{p d}(\hat{s})$ from (39), and $\gamma$ from (40). In this case, we get
$H_{p d}(0)=0.0333$ and $\gamma=4.26$. Since $\gamma>2 h=3$, we are able to choose $\alpha=2$ in (41). The PID-controller in (42) gives the closed-loop poles at $-1.91+i 2.91,-1.91-$ $i 2.91,-2.48,-4.85+i 23.17,-4.85-i 23.17,-20$, which satisfy the given $h=1.5$.

Example 3.2: Given the plant transfer function

$$
G(s)=\frac{(s-50)\left(s^{2}+8 s+32\right)}{(s+2)(s-8)\left(s^{2}+12 s+40\right)},
$$

it is an unstable plant with two RHP zeros at $z_{1}=50$ and $z_{2}=\infty$. We use Proposition 2 to design a PID-controller.

Consider $h=1.5$. Choose arbitrarily $k_{2}=10$, and $a_{1}=$ $a_{2}=3.1$ to make sure that they are greater than $h$. We have to choose $\alpha=\beta=0.01$ carefully, so that they satisfy the constraints in (24) through (27). In this case, the left side of (27), which equals to 0.0633 , is less than the right side of (27), which equals to 0.0833 . From (30), we get the PDcontroller. The closed-loop poles are $-4.44+i 6.00,-4.44-$ $i 6.00,-8.47+i 8.75,-8.47-i 8.75,-113.514$, which satisfy the given $h=1.5$.

To add the I-controller from Proposition 3, we follow the same procedure as that in example 3.1. The PID-controller in (42) gives the closed-loop poles at $-2.30+i 9.55,-2.30-$ $i 9.55,-4.95+i 1.34,-4.95-i 1.34,-11.44-113.40$, which satisfy the given $h=1.5$.

\section{DISCUSSION}

For a class of stable or unstable MIMO plants, we obtained sufficient conditions for the existence of PID-controllers that achieve integral-action and their closed-loop poles with real-parts less than $-h$. We proposed systematic design procedures to design such controllers, which allow freedom in the choice of parameters. How to use these parameters to improve system's performance is a challenging task. Future direction of this study will also include possible extension to more classes of plants.

\section{REFERENCES}

[1] A. N. Gündeş, A. B. Özgüler, "PID stabilization of MIMO plants," IEEE Transactions on Automatic Control, to appear.

[2] T. S. Chang, A. N. Gündeş, "PID Controller Design with Guaranteed Stability Margin for MIMO Systems," to appear at Proceedings of International Conference on Modeling, Simulation and Control, October, 2007.

[3] G. C. Goodwin, S. F. Graebe, M. E. Salgado, Control System Design, Prentice Hall, New Jersey, 2001.

[4] M. Vidyasagar, Control System Synthesis: A Factorization Approach, MIT Press, 1985.

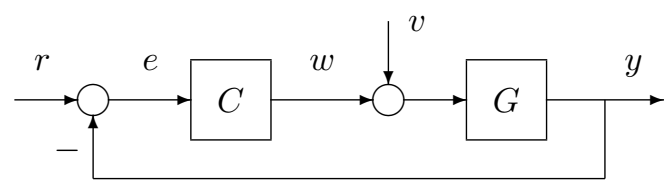

Fig. 1. Unity-Feedback System $\operatorname{Sys}(G, C)$. 